\title{
Quantitative volume and dynamic contrast-enhanced MRI derived perfusion of the infrapatellar fat pad in patellofemoral pain
}

\author{
Rianne A. van der Heijden ${ }^{1 \#}$, Bas A. de Vries ${ }^{1 \#}$, Dirk H. J. Poot ${ }^{1}$, Marienke van Middelkoop ${ }^{2}$, \\ Sita M. A. Bierma-Zeinstra ${ }^{2}$, Gabriel P. Krestin ${ }^{1}$, Edwin H. G. Oei ${ }^{1}$ \\ ${ }^{1}$ Department of Radiology \& Nuclear Medicine, ${ }^{2}$ Department of General Practice, Erasmus MC, Rotterdam, The Netherlands \\ "These authors contributed equally to this work.
}

Correspondence to: Edwin H. G. Oei, MD, PhD. Department of Radiology \& Nuclear Medicine, Erasmus MC, Room Nd-547, P.O. Box 2040, 3000

CA, Rotterdam, The Netherlands. Email: e.oei@erasmusmc.nl.

\begin{abstract}
Background: Patellofemoral pain (PFP) is a common knee condition and possible precursor of knee osteoarthritis (OA). Inflammation, leading to an increased perfusion, or increased volume of the infrapatellar fat pad (IPFP) may induce knee pain. The aim of the study was to compare quantitative dynamic contrastenhanced magnetic resonance imaging (DCE-MRI) parameters, as imaging biomarkers of inflammation, and volume of the IPFP between patients with PFP and controls and between patients with and without IPFP edema or joint effusion.
\end{abstract}

Methods: Patients with PFP and healthy controls were included and underwent non-fat suppressed 3D fast-spoiled gradient-echo (FSPGR) and DCE-MRI. Image registration was applied to correct for motion. The IPFP was delineated on FSPGR using Horos software. Volume was calculated and quantitative perfusion parameters were extracted by fitting extended Tofts' pharmacokinetic model. Differences in volume and DCE-MRI parameters between patients and controls were tested by linear regression analyses. IPFP edema and effusion were analyzed identically.

Results: Forty-three controls and 35 PFP patients were included. Mean IPFP volume was $26.04(4.18) \mathrm{mL}$ in control subjects and $27.52(5.37) \mathrm{mL}$ in patients. Median $\mathrm{K}_{\text {trans }}$ was $0.017(0.016) \mathrm{min}^{-1}$ in control subjects and $0.016(0.020) \mathrm{min}^{-1}$ in patients. None of the differences in volume and perfusion parameters were statistically significant. Knees with effusion showed a higher perfusion of the IPFP compared to knees without effusion in patients only.

Conclusions: The IPFP has been implicated as source of knee pain, but higher DCE-MR blood perfusion, an imaging biomarker of inflammation, and larger volume are not associated with PFP. Patient's knees with effusion showed a higher perfusion, pointing towards inflammation.

Keywords: Knee; patellofemoral pain syndrome (PFP syndrome); inflammation; magnetic resonance imaging (MRI); perfusion

Submitted Mar 13, 2020. Accepted for publication Jul 21, 2020.

doi: $10.21037 /$ qims-20-441

View this article at: http://dx.doi.org/10.21037/qims-20-441

\section{Introduction}

Patellofemoral pain (PFP) is a common knee disorder in active young individuals comprising pain in and around the kneecap. Symptoms commonly occur during knee loading activities, such as running and stair climbing, and during sitting with the knees bent (1). Despite of a variety of treatment options, such as exercise therapy, patellar taping/bracing and foot orthoses, a large group of patients with persistent complaints remains (2-5). PFP has been 
implicated as precursor of knee osteoarthritis (OA), but the exact pathophysiology remains unknown (6-8).

Pathophysiologic processes of the infrapatellar fat pad (IPFP), also known as 'Hoffa's fat pad', have been proposed as a possible source of knee pain (9-13). The IPFP is a richly innervated, highly vascularized, intracapsular, extra-synovial structure in the anterior knee joint between the patella and femur, where it plays a biomechanical role (12,14-16). Structural changes of the IPFP, for example focal IPFP edema as sign of inflammation, have been pinpointed as precursor for structural knee OA, and a larger IPFP size was found in patients with patellofemoral OA (PFOA) (17-19). IPFP size could increase as a result of low-grade inflammation due to repetitive mechanical overload, as for example in Hoffa disease (20) or, hypothetically, can also be larger to begin with and predispose to pain without any pathophysiologic cause. In a PFP population no differences in the presence of focal edema of the IPFP between healthy control subjects and patients with PFP was demonstrated (21). IPFP size has been studied in OA $(17,22)$, but has not been studied in PFP yet. Besides the biomechanical role, it is also suggested that the IPFP is an osteoarthritic joint tissue capable of modulating inflammatory responses in knee OA (11). This might also apply to PFP.

Dynamic contrast-enhanced magnetic resonance imaging (DCE-MRI) enables non-invasive evaluation of inflammation by measuring blood perfusion, which is known to increase in the presence of inflammation. DCE-MRI derived increased blood perfusion parameters are therefore considered imaging biomarkers of inflammation in various musculoskeletal tissues (23-28). To our knowledge, only one prior study applied semi-quantitative DCE-MRI in the IPFP of obese patients with knee $\mathrm{OA}$ and showed a correlation between knee pain and inflammation (28). In a previous study including the same study population as the current study, quantitative DCE-MRI analysis of the patella identified an increased patellar perfusion, contrary to the decreased patellar perfusion based on vascular alterations that was expected (29). Among the potential explanations of increased perfusion is the occurrence of an inflammatory process in which the IPFP plays an important modulating role (12).

Therefore, the aim of this study was to compare quantitative DCE-MRI blood perfusion parameters, as imaging biomarkers of inflammation, and volume of the IPFP between patients with PFP and healthy control subjects. A second aim was to explore if specific perfusion patterns exist in patients based on correlation of DCEMRI perfusion parameters with clinical or MR imaging characteristics that potentially are related to inflammation or perfusion. Hypothetically, a larger IPFP volume and higher blood perfusion values are expected in patients with PFP than in healthy control subjects.

\section{Methods}

\section{Study design and participants}

In the current study, data was analyzed from a previously conducted cross sectional case-control study. Patients with minimum symptom duration of 2 months to a maximum of 2 years and healthy control subjects were included between January 2013 and September 2014. Patients who visited their general practitioner, physiotherapist or sports physician were included if diagnosed with PFP based on the presence of at least three of the following symptoms: pain while stair climbing; while squatting; while running; while cycling; while sitting for a prolonged period with the knee flexed, or crepitus. Exclusion criteria were: previous PFP episodes more than 2 years ago, onset after trauma, defined pathological condition of the affected knee at present, or previous surgery or injury of the affected knee. Healthy controls were recruited from patients' sports team members, friends, or colleagues. Exclusion criteria of controls were: history of PFP, surgery or injury of both knees, or firstdegree relatedness with patients. Other exclusion criteria for both groups were: contra-indications for contrast-enhanced MRI and insufficient knowledge of the Dutch language. Patients and controls were aimed to match for age, gender, body mass index (BMI), and activity level. Full details of this study have been published elsewhere (21). This study was approved by our institutional review board, is conducted in accordance with the Helsinki Declaration and written informed consent was obtained from all participants. All patients and controls, aged 18-40 years, with DCE-MRI data available were included in the current analysis.

\section{Image acquisition and measures}

Participants underwent 3 Tesla MRI (Discovery MR750, GE Healthcare, USA) using a dedicated 8-channel knee coil (Invivo Inc., USA) at our institution. One knee, the (most) symptomatic knee of PFP patients was selected, or randomly chosen if both knees were equally painful or if both were asymptomatic (controls). The MRI protocol consisted of routine clinical proton density and T2weighted fat-saturated sequences in three orthogonal planes, 


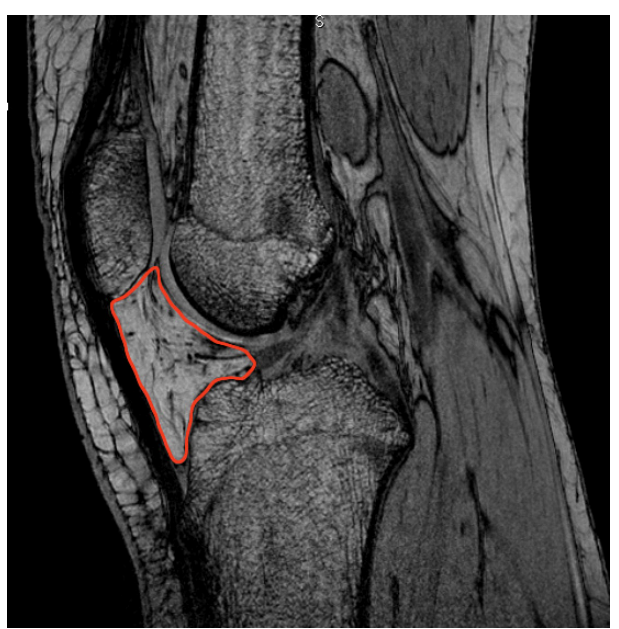

Figure 1 Delineation of the IPFP on the non-FS FSPGR sequence (red line). IPFP, infrapatellar fat pad; non-FS FSPGR, non-fatsaturated fast-spoiled gradient-echo.

and a sagittal 3D non-fat-saturated fast-spoiled gradientecho (non-FS FSPGR) sequence with a slice thickness of $0.5 \mathrm{~mm}$.

DCE-MRI was acquired by a time-resolved imaging of contrast kinetics (TRICKS) sequence with anterior-posterior (AP) frequency encoding direction to avoid pulsation artifacts of the popliteal artery into the region of interest. MRI parameters were: in-plane pixel resolution $1.5 \mathrm{~mm}$, slice thickness $5 \mathrm{~mm}$, field of view $380 \times 380 \times 70 \mathrm{~mm}$, acquisition matrix $256 \times 128,14$ sagittal slices, $70 \%$ sampling in the phase direction, $\mathrm{TE}=1.7 \mathrm{~ms}, \mathrm{TR}=9.3 \mathrm{~ms}, \mathrm{FA}=30^{\circ}$. The DCEMRI protocol consisted of 35 phases of $10.30 \pm 0.07 \mathrm{~s}$ (constant within subject). Intravenous contrast administration of $0.2 \mathrm{mmol} / \mathrm{kg}$ gadopentetate dimeglumine (Magnevist, Bayer, Berlin, Germany), at a rate of $2 \mathrm{~mL} / \mathrm{s}$, was started after the first phase. Additionally, a non-fat-suppressed 3D FSPGR sequence with in-plane resolution of $0.3 \mathrm{~mm} \times 0.3 \mathrm{~mm}$ and $0.5 \mathrm{~mm}$ slices was acquired before contrast administration for delineation of the patellar bone marrow.

In addition, participants completed a questionnaire on demographics, sports participation (yes/no) and knee complaints [Numerical Rating Score (NRS) pain score during rest and exercise, duration of symptoms, and function measured by the Anterior Knee Pain Scale (AKPS) 0-100 (30)]. Finally, a physical examination was performed in which the pressure pain threshold at the contralateral arm was tested as a measure of pain sensitization, according to a prior published method $(31,32)$.

\section{Image analysis}

IPFP edema located centrally and superolaterally and joint effusion were already assessed as part of the Magnetic Resonance Imaging Osteoarthritis Knee Score (MOAKS) with several additional scoring items in a prior study $(21,33)$.

The volume of interest (VOI) consisted of the whole IPFP with the following boundaries according to a recent study: the inferior patellar pole, femoral intercondylar notch, proximal patellar tendon, intermeniscal ligament, both menisci and the anterior tibia (15). VOIs were delineated in correspondence with the DCE-MRI data on the nonFS FSPGR sequence, which has previously been reported to be superior to fat-saturated images (Figure 1) (34). All VOIs were drawn by a senior radiology resident subspecializing in musculoskeletal imaging (RA van der Heijden) after careful consideration of the boundaries in the first 10 subjects together with a senior musculoskeletal radiologist (EHGO). DCE-MRI time points were registered using an automated rigid body registration with Elastix (35). Horos software (Horosproject.org, USA) was used to delineate the VOIs, register the non-FS FSPGR and DCE-maps, calculate the $3 \mathrm{D}$ volume and extract the perfusion parameters of the VOI with the DCE-tool (36). Fitting a pharmacokinetic model to the data enables extraction of quantitative DCE-MRI parameters which (to a greater or lesser extent) reflect physiological phenomena such as blood flow, blood volume, and extravascular permeability (37). Tofts' pharmacokinetic model has shown to be the most accurate model for patellar bone (38). Tofts' extended model is more suitable for highly vascularized structures, such as the IPFP, by adding the vascular term $V_{p}$ (37). The arterial input function (AIF) was estimated in each participant using a ROI in the popliteal artery. All fitted AIF's were visually checked. Quantitative DCE-MRI perfusion parameters $\mathrm{K}_{\text {trans }}, \mathrm{K}_{\mathrm{ep}}, \mathrm{V}_{\mathrm{e}}$ and $\mathrm{V}_{\mathrm{p}}$ ) were extracted by fitting the Tofts' extended pharmacokinetic model (Figure 2) (39). $\mathrm{K}_{\text {trans }}$ reflects the volume transfer constant into the tissue compartment, $\mathrm{K}_{\mathrm{ep}}$ describes the rate constant back into the vascular component, $\mathrm{V}_{\mathrm{e}}$ the extravascular extracellular space and $V_{p}$ the vascular fraction.

\section{Statistical analysis}

The Shapiro-Wilk test was used to evaluate normality of the distribution of the parameters. Independent sample $t$-tests and chi-square tests, or Mann-Whitney $\mathrm{U}$ tests if data distribution was not normal, were applied to investigate 


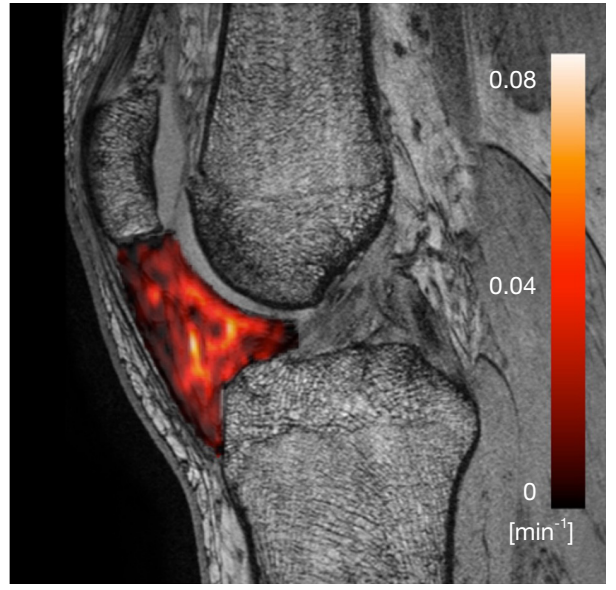

Figure 2 Overlay of a $K_{\text {trans }}$ map of the IPFP on the non-FS FSPGR sequence in a PFP patient. IPFP, infrapatellar fat pad; non-FS FSPGR, non-fat-saturated fast-spoiled gradient-echo; PFP, patellofemoral pain.

differences in baseline characteristics between groups.

The DCE image analysis resulted in a mean value for the perfusion parameters within each VOI. The mean and standard deviation over all subjects were calculated for control subjects and PFP patients separately. Differences in variance of volume and perfusion parameters across subjects were tested with Levene's test. Volume followed a normal distribution. All perfusion parameters showed a normal distribution of residuals after logarithmic transformation and, accordingly, regression analyses could be performed. Differences in volume and perfusion parameters between groups were compared by linear regression analyses, adjusted for age, gender, BMI, and sports participation. Furthermore, possible patient subgroups were explored by multivariate linear regression analysis of the following variables: pain during rest and during exercise, duration of complaints, presence of sitting pain, function, pain pressure threshold contralateral arm as sensitization measure, presence of IPFP edema centrally and superolaterally and joint effusion. $\mathrm{P}$ values $<0.05$ were considered to be statistically significant for the main linear regression analyses. Results are presented as mean differences with $95 \%$ confidence intervals. For the subgroup' analyses, a lower $\mathrm{P}$ value of $<0.01$ was applied due to multiple testing. All analyses were performed with SPSS v25 (IBM, USA).

\section{Results}

\section{Population and patient characteristics}

In a prior study, 64 patients with $\mathrm{PFP}$ and 70 control subjects aged 14-40 years were included (Figure 3). DCEMRI was only acquired in adults and image quality was sufficient in 35 adults PFP patients and 44 adult control subjects. Mean age was 26.1 (range, 18-40, SD 5.0) years, mean BMI was 24.1 (SD, 3.4) $\mathrm{kg} / \mathrm{m}^{2}$ and $49 \%$ (39) was female. The BMI was significantly higher in the patient group (Table 1). Patients reported a mean duration of complaints of 11.2 months and $45.7 \%$ (16) reported bilateral pain. Centrally located moderate to severe IPFP edema was present in one patient and two control subjects. Superolateral IPFP edema was present in 16 patients and 19 control subjects. Medium to large effusion (corresponding with MOAKS grade 2-3) was present in four patients and seven control subjects. There were no significant differences in the presence of these features.

\section{Volume and DCE-MRI parameters}

Due to a lack of a plateau phase in the time intensity curve, the fitting algorithm might not provide valid values of $\mathrm{V}_{\mathrm{e}}$ and subsequently $\mathrm{K}_{\mathrm{ep}}$ and therefore those parameters were not shown.

Mean IPFP volume was 26.04 (4.18) $\mathrm{mL}$ in control subjects and $27.52(5.37) \mathrm{mL}$ in patients. Median $\mathrm{K}_{\text {trans }}$ was $0.017(0.016) \mathrm{min}^{-1}$ in control subjects and 0.016 (0.020) min $^{-1}$ in patients. Median $\mathrm{V}_{\mathrm{p}}$ was remarkably higher in patients than control subjects, respectively 0.00037 $(0.00039)$ and $0.00029(0.00033)$, but this difference was not statistically significant $(\mathrm{P}=0.10)$. Mean volume and perfusion parameters did not differ between groups (Table 2, Figure 3).

$V_{p}$ was the only perfusion parameter for which Levene's test showed a significant difference in variance across groups $(\mathrm{P}=0.035)$. Further visual inspection of the boxplots showed two outliers in the patient group, which most likely account for the difference in variance (Figure S1: boxplot without the largest patient outlier).

The patient subgroup analyses showed that presence of joint effusion was significantly associated with elevated $\mathrm{K}_{\text {trans }}(\mathrm{P}=0.002)$ and $\mathrm{V}_{\mathrm{p}}(\mathrm{P}=0.004)$, but not with volume $(\mathrm{P}=0.41)$. Remarkably, this association was not present in control subjects. None of the other subgroup analyses 

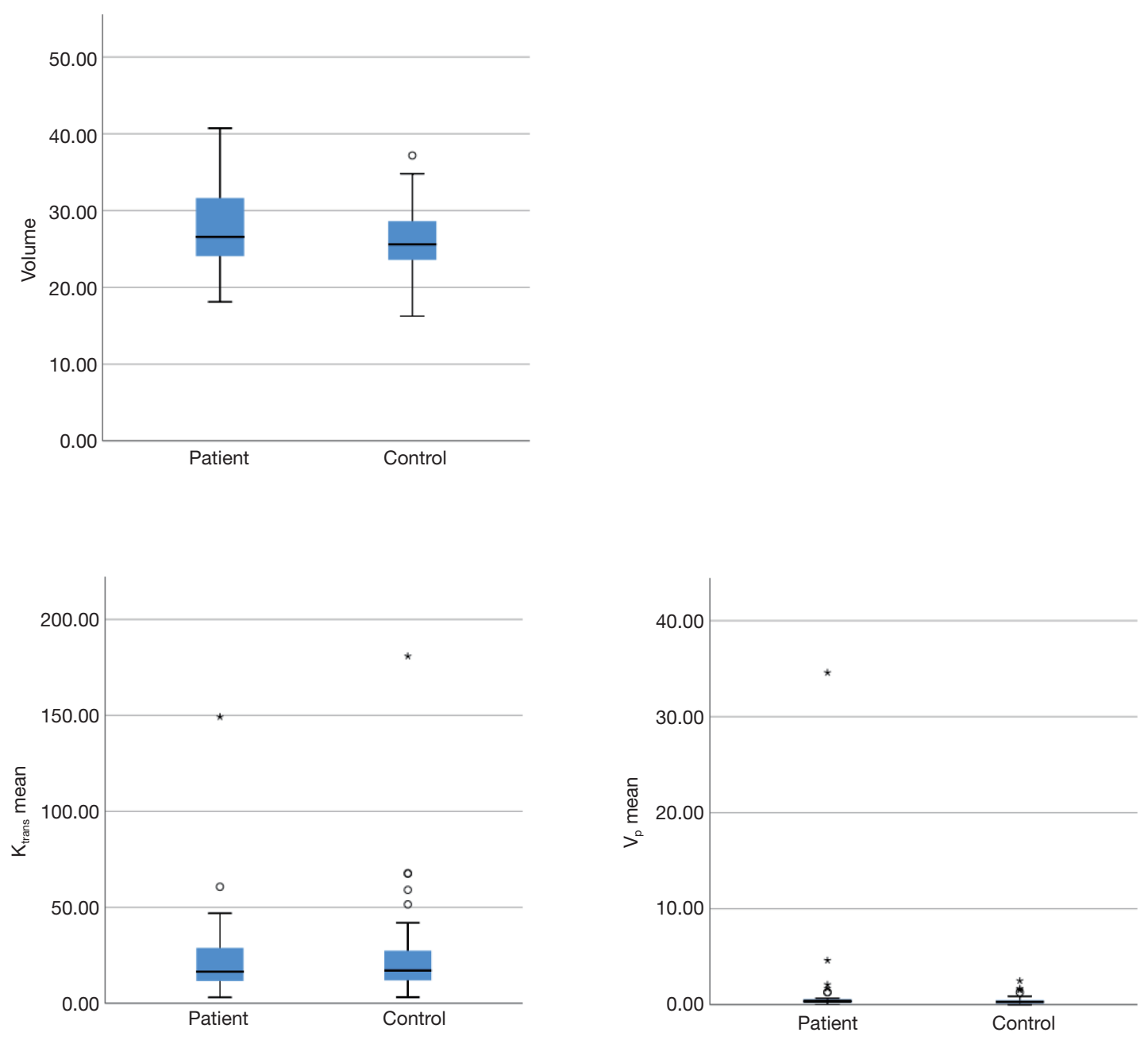

Figure 3 Boxplots of volume $(\mathrm{mL})$ and perfusion parameters $\mathrm{K}_{\text {trans }}\left(\mathrm{min}^{-1}\right)$ and $\mathrm{V}_{\mathrm{p}}$ (multiplied by 1,000$)$. The * means the outliers.

revealed significant associations for respectively volume/ $\mathrm{K}_{\text {trans }} / \mathrm{V}_{\mathrm{p}}$ : pain during rest $\mathrm{P}=0.76 / 0.74 / 0.31$; during exercise $\mathrm{P}=0.07 / 0.25 / 0.51$; duration of complaints $\mathrm{P}=0.78 / 0.30 / 0.14$; presence of sitting pain $\mathrm{P}=0.29 / 0.23 / 0.24$; function $\mathrm{P}=0.57 / 0.88 / 0.48$; pain pressure threshold contralateral arm as sensitization measure $\mathrm{P}=0.82 / 0.54 / 0.27$; presence of IPFP edema centrally $\mathrm{P}=0.11 / 0.44 / 0.56$; presence of IPFP edema superolaterally $\mathrm{P}=0.82 / 0.75 / 0.54$.

\section{Discussion}

In this study, volume and quantitative DCE-MRI blood perfusion parameters $K_{\text {trans }}$ and $V_{p}$ of the IPFP were compared between healthy control subjects and patients with PFP. In contrast to our hypothesis, no significant differences were found in volume or DCEMR blood perfusion parameters, as imaging biomarkers of inflammation, of the IPFP between healthy control subjects and patients with PFP. Furthermore, patient subgroups were explored in search of an association of DCE-MRI parameters with clinical characteristics and MR features potentially related to volume or perfusion/inflammation. Only effusion was significantly associated with higher $\mathrm{K}_{\text {trans }}$ and $V_{p}$ in patients with PFP.

In recent studies, inconsistent results were found in $\mathrm{OA}$ populations regarding IPFP volume. One study found an association between a larger volume and fewer structural abnormalities in patients with clinical knee OA, suggesting a protective role of a larger IPFP (40). Two other studies did not find a correlation between IPFP volume and 
Table 1 Characteristics of study participants

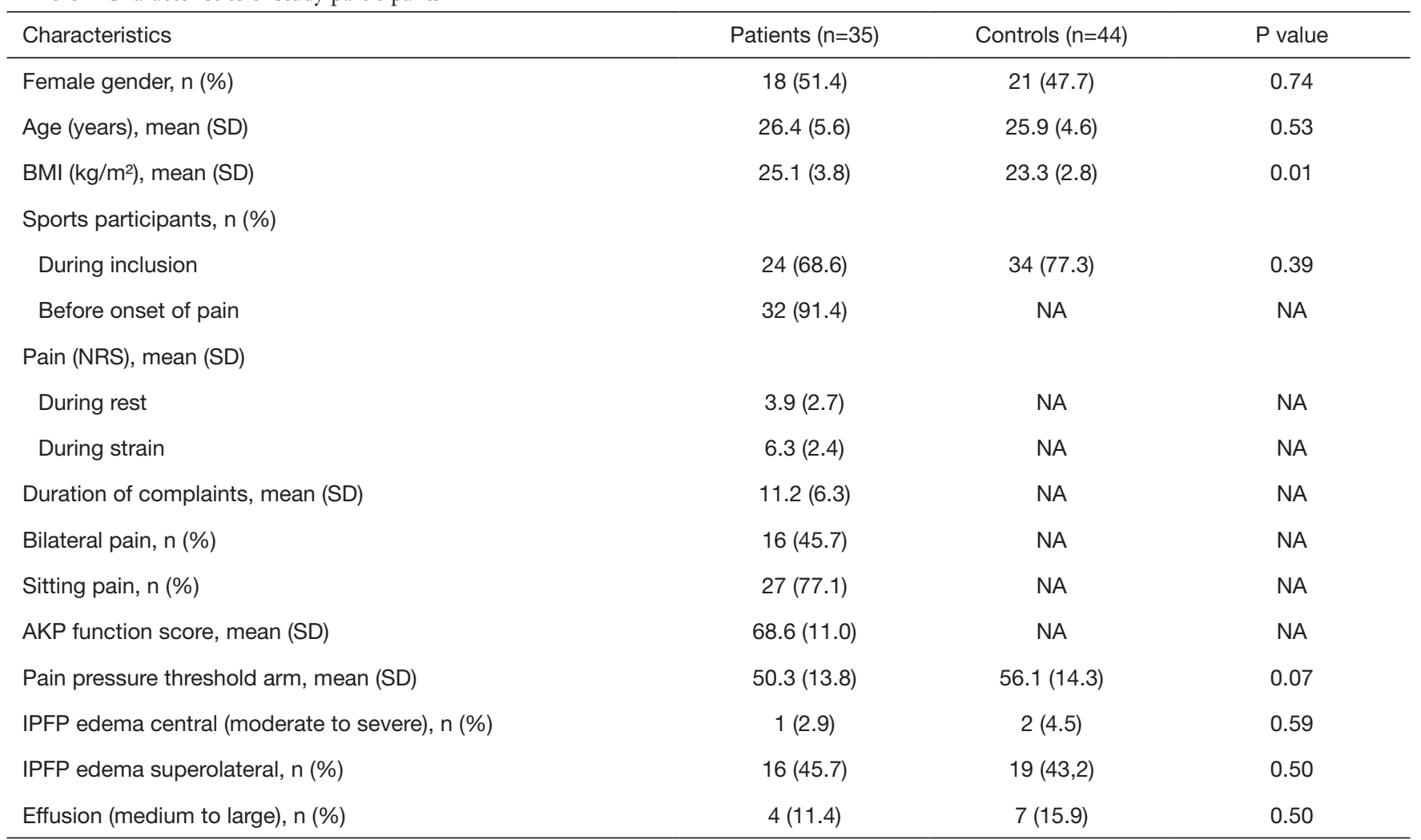

BMI, body mass index; NRS, Numerical Rating Score; AKP, Anterior Knee Pain; IPFP, infrapatellar fat pad.

Table 2 Mean (SD) of the volume and median (IQR) of the VOI mean for $\mathrm{K}_{\text {trans }}$ and $\mathrm{V}_{\mathrm{p}}$ and mean difference (95\% CI) and adjusted $\mathrm{P}$ value in patients and controls

\begin{tabular}{lcccc}
\hline Measures & Patients $(\mathrm{n}=35)$ & Controls $(\mathrm{n}=44)$ & Mean difference $(95 \%$ Cl $)$ & Adjusted P value \\
\hline Volume $(\mathrm{mL})$ & $27.52(5.37)$ & $26.04(4.18)$ & $1.48(-0.66,3.62)$ & 0.07 \\
$\mathrm{~K}_{\text {trans }}\left(\mathrm{min}^{-1}\right)$ & $0.016(0.020)$ & $0.017(0.016)$ & $-1.12(-13.47,11.21)$ & 0.98 \\
$\mathrm{~V}_{\mathrm{p}}$ & $0.00037(0.00039)$ & $0.00029(0.00033)$ & $1.10(-0.65,2.85)$ & 0.10 \\
\hline
\end{tabular}

VOI, volume of interest.

symptomatic or radiographic knee OA $(17,41)$. A fourth study, specifically focusing on PFOA, demonstrated that individuals with PFOA had a larger IPFP than controls, and a larger IPFP volume was directly related to pain (42) Since age presumably influences fat pad volume in patient with OA, the current volumes cannot be directly compared with the prior study including an older population of patients with PF OA (43).

The single prior study that applied DCE-MRI, in a semi-quantitative manner, to investigate IPFP inflammation found a relation between their perfusion derived inflammation marker and knee pain in obese patients with knee OA (28). DCE-MRI had not yet been applied in the IPFP of patients with PFP and healthy control subjects.

The lack of a difference between patients with PFP and control subjects might be explained by a still preserved tissue homeostasis in PFP without induced inflammatory response. In order to determine if a certain patient subgroup with explicit inflammation could be identified, additional exploratory subgroup analyses were conducted focusing on clinical characteristics and MR features potentially related inflammation. For instance, a larger size and higher 
perfusion of the IPFP could be expected in patients with other proposed signs of inflammation, such as Hoffa edema or effusion. Furthermore, we hypothesized that low-grade inflammation resulting in higher perfusion and/or increased volume would be associated with more pain and worse function or would have a more systemic effect resulting in longer symptom duration or presence of sensitization. Finally, an increase in size or vascular changes could also have been the answer to the enigma of patients with PFP, which explicitly exhibit pain during sitting with the knees bent $(29,44,45)$. In the end, none of these variables were significantly associated with volume or perfusion parameters besides effusion. Effusion was significantly associated with $\mathrm{K}_{\text {trans }}$ and $\mathrm{V}_{\mathrm{p} .}$ indicating a larger vascular fraction and more blood inflow in patients. Further evaluation revealed that these associations were not present in control subjects.

This implies that joint effusion is only associated with inflammation and neovascularization in patients with PFP. These results are only speculative, though, due to the low number of subjects per group. A potential explanation for the difference between groups might lie in a different mechanism of effusion, implying an inflammatory pathway in patients only. In control subjects one possible explanation might be a mechanical pathway.

In this study, for the first time, the IPFP volume and IPFP blood perfusion were quantitatively analyzed by DCE-MRI in order to unravel the role of the IPFP in the pathophysiology of PFP. A strength of this study is the inclusion of healthy control subjects next to patients with PFP. Furthermore, the current study applied quantitative assessment of DCEMRI perfusion values, which offers more robust parameters that directly represent the microvasculature physiology, in contrast to semi-quantitative analysis.

A potential limitation might be the lack of $\mathrm{B} 1+$ or precontrast T1 map, which led to the use of a literature based fixed T1(0) value of $1,443 \mathrm{~ms}$. We do not expect this to have affected the outcome, since no differences in native T1 variability were expected between groups. Furthermore, a dedicated transmit/receive knee coil with relatively homogeneous B1 field was used. Second, quality of DCEMRI imaging data was not sufficient in nine patients and six control subjects due to artifacts at the beginning of the study. We do not expect this to have influenced our conclusions, as the baseline characteristics of these participants did not differ from the participants in which the DCE-MRI was sufficient. Due to time constraints in our MR protocol, DCE-MRI acquisition did not last long enough for the time intensity curve to reach plateau phase.
As this might potentially lead to unreliable estimates of $\mathrm{V}_{\mathrm{e}}$ and subsequently also of $\mathrm{K}_{\mathrm{ep}}$, only the robust parameters $K_{\text {trans }}$ and $V_{p}$ were presented. This is sufficient for our research purpose as these two are the most important parameters to identify increased perfusion.

ROI delineation was done by a single observer only, which leaves the variability introduced by having an alternative observer unknown. A previous study stated that the inter-observer variability is low when a large ROI is used (46). This was done in quantitative MRI of cartilage, but given our experience in both cartilage and other structures like the fat pad we found these comparable. Furthermore, the boundaries of the ROI were discussed in depth with a senior musculoskeletal radiologist (EHGO).

Since a group-wise AIF is not available within the DCEtool, we used a subject-specific AIF, despite the probability of not capturing the arterial bolus given the low temporal resolution of 10 seconds. Therefore, all AIF curves were visually checked and appeared to capture the bolus peak adequately. Another point to notice is that small, yet significant differences could have been left undetected due to the large inter-subject variability. Power analysis was not feasible in advance, due to the lack of knowledge regarding effect sizes. In future research including a larger number of subjects would be advised in order to be able to draw firm conclusions. With regard to our results, we do not think this would affect the main outcome as inter-subject variability is equally present in both groups and the variance was not statistically significantly different between groups, except for $V_{p}$. The clinical relevance of these potentially missed small differences would also be questionable given the small effect sizes and the fact that patients had a lower mean of $\mathrm{K}_{\text {trans }}$ and $\mathrm{V}_{\mathrm{p}}$, which does not concur with the suspected increased blood perfusion accompanying inflammation. Finally, some of the subgroup analyses were theoretically underpowered due to the low prevalence of features. If these would have been the key features for the incidence of PFP, though, a higher prevalence would have been expected to start with. In general, due to the cross-sectional design no causal relations could be studied, but only associations. Future research might try to achieve a complete picture of the perfusion in the knee joint. For now, this is quite challenging, because different tissues require different pharmacokinetic models.

In conclusion, patients with PFP and healthy control subjects do not demonstrate a significantly different volume or blood perfusion of the IPFP. Thus, it seems that higher IPFP blood perfusion measured by DCE-MRI, as imaging 
biomarker of inflammation, and larger volume are not associated with PFP.

\section{Acknowledgments}

We would like to acknowledge Kyung Hyun Sung for the development of the DCE tool in Horos.

Funding: This research was partially funded by a Young Researchers Grant awarded by the European Society of Musculoskeletal Radiology, a seed grant from the Radiological Society of North America (RSNA), an EUR fellowship from the Erasmus University Rotterdam and the Dutch Arthritis Foundation.

\section{Footnote}

Conflicts of Interest: All authors have completed the ICMJE uniform disclosure form (available at http://dx.doi. org/10.21037/qims-20-441). EHGO serves as an unpaid Associate Editor of Quantitative Imaging in Medicine and Surgery. Dr. RA van der Heijden reports grants from European Society Skeletal Radiology, during the conduct of the study; Dr. M van Middelkoop reports grants from Erasmus University Rotterdam, during the conduct of the study; Dr. SMABZ reports grants from Dutch Artritis Foundation, during the conduct of the study; Dr. EHGO reports grants from RSNA, during the conduct of the study. The other authors have no conflicts of interest to declare.

Ethical Statement: The study was approved by the institutional ethics committee of the Erasmus University Medical Center (No. 2012-342). Written informed consent was obtained from the patient for publication of this study and any accompanying images.

Open Access Statement: This is an Open Access article distributed in accordance with the Creative Commons Attribution-NonCommercial-NoDerivs 4.0 International License (CC BY-NC-ND 4.0), which permits the noncommercial replication and distribution of the article with the strict proviso that no changes or edits are made and the original work is properly cited (including links to both the formal publication through the relevant DOI and the license). See: https://creativecommons.org/licenses/by-nc-nd/4.0/.

\section{References}

1. Crossley KM, Stefanik JJ, Selfe J, Collins NJ, Davis IS,
Powers CM, McConnell J, Vicenzino B, Bazett-Jones DM, Esculier JF, Morrissey D, Callaghan MJ. 2016 Patellofemoral pain consensus statement from the 4th International Patellofemoral Pain Research Retreat, Manchester. Part 1: Terminology, definitions, clinical examination, natural history, patellofemoral osteoarthritis and patient-reported outcome measures. Br J Sports Med 2016;50:839-43.

2. van der Heijden RA, Lankhorst NE, van Linschoten R, Bierma-Zeinstra SM, van Middelkoop M. Exercise for treating patellofemoral pain syndrome. Cochrane Database Syst Rev 2015;1:CD010387.

3. Callaghan MJ, Selfe J. Patellar taping for patellofemoral pain syndrome in adults. Cochrane Database Syst Rev 2012;4:CD006717.

4. Barton CJ, Munteanu SE, Menz HB, Crossley KM. The efficacy of foot orthoses in the treatment of individuals with patellofemoral pain syndrome: a systematic review. Sports Med 2010;40:377-95.

5. Swart NM, van Linschoten R, Bierma-Zeinstra SM, van Middelkoop M. The additional effect of orthotic devices on exercise therapy for patients with patellofemoral pain syndrome: a systematic review. Br J Sports Med 2012;46:570-7.

6. Thomas MJ, Wood L, Selfe J, Peat G. Anterior knee pain in younger adults as a precursor to subsequent patellofemoral osteoarthritis: a systematic review. BMC Musculoskelet Disord 2010;11:201.

7. Eijkenboom JFA, Waarsing JH, Oei EHG, BiermaZeinstra SMA, van Middelkoop M. Is patellofemoral pain a precursor to osteoarthritis?: Patellofemoral osteoarthritis and patellofemoral pain patients share aberrant patellar shape compared with healthy controls. Bone Joint Res 2018;7:541-7.

8. Crossley KM, Hinman RS. The patellofemoral joint: the forgotten joint in knee osteoarthritis. Osteoarthritis Cartilage 2011;19:765-7.

9. Biedert RM, Sanchis-Alfonso V. Sources of anterior knee pain. Clin Sports Med 2002;21:335-47, vii.

10. Dye SF. The pathophysiology of patellofemoral pain: a tissue homeostasis perspective. Clin Orthop Relat Res 2005;(436):100-10.

11. Felson DT. The sources of pain in knee osteoarthritis. Curr Opin Rheumatol 2005;17:624-8.

12. Clockaerts S, Bastiaansen-Jenniskens YM, Runhaar J, Van Osch GJ, Van Offel JF, Verhaar JA, De Clerck LS, Somville J. The infrapatellar fat pad should be considered as an active osteoarthritic joint tissue: a narrative review. 
Osteoarthritis Cartilage 2010;18:876-82.

13. Ioan-Facsinay A, Kloppenburg $M$. An emerging player in knee osteoarthritis: the infrapatellar fat pad. Arthritis Res Ther 2013;15:225.

14. Dragoo JL, Johnson C, McConnell J. Evaluation and treatment of disorders of the infrapatellar fat pad. Sports Med 2012;42:51-67.

15. Stephen JM, Sopher R, Tullie S, Amis AA, Ball S, Williams A. The infrapatellar fat pad is a dynamic and mobile structure, which deforms during knee motion, and has proximal extensions which wrap around the patella. Knee Surg Sports Traumatol Arthrosc 2018;26:3515-24.

16. Pan F, Han W, Wang X, Liu Z, Jin X, Antony B, Cicuttini F, Jones G, Ding C. A longitudinal study of the association between infrapatellar fat pad maximal area and changes in knee symptoms and structure in older adults. Ann Rheum Dis 2015;74:1818-24.

17. Ruhdorfer A, Haniel F, Petersohn T, Dorrenberg J, Wirth W, Dannhauer T, Hunter DJ, Eckstein F. Betweengroup differences in infra-patellar fat pad size and signal in symptomatic and radiographic progression of knee osteoarthritis vs non-progressive controls and healthy knees - data from the FNIH Biomarkers Consortium Study and the Osteoarthritis Initiative. Osteoarthritis Cartilage 2017;25:1114-21.

18. Han W, Aitken D, Zhu Z, Halliday A, Wang X, Antony B, Cicuttini F, Jones G, Ding C. Signal intensity alteration in the infrapatellar fat pad at baseline for the prediction of knee symptoms and structure in older adults: a cohort study. Ann Rheum Dis 2016;75:1783-8.

19. Wang K, Ding C, Hannon MJ, Chen Z, Kwoh CK, Hunter DJ. Quantitative Signal Intensity Alteration in Infrapatellar Fat Pad Predicts Incident Radiographic Osteoarthritis: The Osteoarthritis Initiative. Arthritis Care Res (Hoboken) 2019;71:30-8.

20. Roemer FW, Jarraya M, Felson DT, Hayashi D, Crema MD, Loeuille D, Guermazi A. Magnetic resonance imaging of Hoffa's fat pad and relevance for osteoarthritis research: a narrative review. Osteoarthritis Cartilage 2016;24:383-97.

21. van der Heijden RA, de Kanter JL, Bierma-Zeinstra SM, Verhaar JA, van Veldhoven PL, Krestin GP, Oei EH, van Middelkoop M. Structural abnormalities on magnetic resonance imaging in patients with patellofemoral pain: a cross-sectional case-control study. Am J Sports Med 2016;44:2339-46.

22. Burda B, Steidle-Kloc E, Dannhauer T, Wirth W, Ruhdorfer A, Eckstein F. Variance in infra-patellar fat pad volume: Does the body mass index matter?-Data from osteoarthritis initiative participants without symptoms or signs of knee disease. Ann Anat 2017;213:19-24.

23. Boesen M, Kubassova O, Bouert R, Axelsen MB, Ostergaard M, Cimmino MA, Danneskiold-Samsoe B, Horslev-Petersen K, Bliddal H. Correlation between computer-aided dynamic gadolinium-enhanced MRI assessment of inflammation and semi-quantitative synovitis and bone marrow oedema scores of the wrist in patients with rheumatoid arthritis--a cohort study. Rheumatology (Oxford) 2012;51:134-43.

24. Axelsen MB, Stoltenberg M, Poggenborg RP, Kubassova O, Boesen M, Bliddal H, Horslev-Petersen K, Hanson LG, Ostergaard M. Dynamic gadolinium-enhanced magnetic resonance imaging allows accurate assessment of the synovial inflammatory activity in rheumatoid arthritis knee joints: a comparison with synovial histology. Scand J Rheumatol 2012;41:89-94.

25. Boesen M, Kubassova O, Sudol-Szopinska I, Maas M, Hansen P, Nybing JD, Oei EH, Hemke R, Guermazi A. MR Imaging of Joint Infection and Inflammation with Emphasis on Dynamic Contrast-Enhanced MR Imaging. PET Clin 2018;13:523-50.

26. Riis RG, Gudbergsen H, Henriksen M, Ballegaard C, Bandak E, Rottger D, Bliddal H, Hansen BB, Hangaard $\mathrm{S}$, Boesen M. Synovitis assessed on static and dynamic contrast-enhanced magnetic resonance imaging and its association with pain in knee osteoarthritis: A crosssectional study. Eur J Radiol 2016;85:1099-108.

27. Riis RG, Gudbergsen H, Simonsen O, Henriksen M, Al-Mashkur N, Eld M, Petersen KK, Kubassova O, Bay Jensen AC, Damm J, Bliddal H, Arendt-Nielsen L, Boesen M. The association between histological, macroscopic and magnetic resonance imaging assessed synovitis in end-stage knee osteoarthritis: a cross-sectional study. Osteoarthritis Cartilage 2017;25:272-80.

28. Ballegaard C, Riis RG, Bliddal H, Christensen R, Henriksen M, Bartels EM, Lohmander LS, Hunter DJ, Bouert R, Boesen M. Knee pain and inflammation in the infrapatellar fat pad estimated by conventional and dynamic contrast-enhanced magnetic resonance imaging in obese patients with osteoarthritis: a cross-sectional study. Osteoarthritis Cartilage 2014;22:933-40.

29. van der Heijden RA, Poot DHJ, Ekinci M, Kotek G, van Veldhoven PLJ, Klein S, Verhaar JAN, Krestin GP, Bierma-Zeinstra SMA, van Middelkoop M, Oei EHG. Blood perfusion of patellar bone measured by dynamic contrast-enhanced MRI in patients with patellofemoral 
pain: a case-control study. J Magn Reson Imaging 2018;48:1344-50.

30. Kujala UM, Jaakkola LH, Koskinen SK, Taimela S, Hurme M, Nelimarkka O. Scoring of patellofemoral disorders. Arthroscopy 1993;9:159-63.

31. van der Heijden RA, Vollebregt T, Bierma-Zeinstra SM, van Middelkoop M. Strength and pain threshold handheld dynamometry test reliability in patellofemoral pain. Int J Sports Med 2015;36:1201-5.

32. van der Heijden RA, Rijndertse MM, Bierma-Zeinstra SMA, van Middelkoop M. Lower pressure pain thresholds in patellofemoral pain patients, especially in female patients: a cross-sectional case-control study. Pain Med 2018;19:184-92.

33. Hunter DJ, Guermazi A, Lo GH, Grainger AJ, Conaghan PG, Boudreau RM, Roemer FW. Evolution of semiquantitative whole joint assessment of knee OA: MOAKS (MRI Osteoarthritis Knee Score). Osteoarthritis Cartilage 2011;19:990-1002.

34. Steidle-Kloc E, Wirth W, Ruhdorfer A, Dannhauer T, Eckstein F. Intra- and inter-observer reliability of quantitative analysis of the infra-patellar fat pad and comparison between fat- and non-fat-suppressed imaging-Data from the osteoarthritis initiative. Ann Anat 2016;204:29-35.

35. Klein S, Staring M, Murphy K, Viergever MA, Pluim JP. elastix: a toolbox for intensity-based medical image registration. IEEE Trans Med Imaging 2010;29:196-205.

36. DCE Tool Plugin. Available online: http://kyungs.bol.ucla. edu/software/DCE_tool/DCE_tool.html (Accessed 23, May 2018).

37. Sourbron SP, Buckley DL. Tracer kinetic modelling in MRI: estimating perfusion and capillary permeability. Phys Med Biol 2012;57:R1-33.

38. Poot DHJ, van der Heijden RA, van Middelkoop M, Oei EHG, Klein S. Dynamic contrast-enhanced MRI of the

Cite this article as: van der Heijden RA, de Vries BA, Poot DHJ, van Middelkoop M, Bierma-Zeinstra SMA, Krestin GP, Oei EHG. Quantitative volume and dynamic contrast-enhanced MRI derived perfusion of the infrapatellar fat pad in patellofemoral pain. Quant Imaging Med Surg 2021;11(1):133-142. doi: 10.21037/qims-20-441 patellar bone: How to quantify perfusion. J Magn Reson Imaging 2018;47:848-58.

39. Tofts PS, Kermode AG. Measurement of the blood-brain barrier permeability and leakage space using dynamic MR imaging. 1. Fundamental concepts. Magn Reson Med 1991;17:357-67.

40. Cai J, Xu J, Wang K, Zheng S, He F, Huan S, Xu S, Zhang H, Laslett L, Ding C. Association between infrapatellar fat pad volume and knee structural changes in patients with knee osteoarthritis. J Rheumatol. 2015;42:1878-84.

41. Steidle-Kloc E, Culvenor AG, Dorrenberg J, Wirth W, Ruhdorfer A, Eckstein F. Relationship between knee pain and infrapatellar fat pad morphology: a within- and between-person analysis from the osteoarthritis initiative. Arthritis Care Res (Hoboken) 2018;70:550-7.

42. Cowan SM, Hart HF, Warden SJ, Crossley KM. Infrapatellar fat pad volume is greater in individuals with patellofemoral joint osteoarthritis and associated with pain. Rheumatol Int 2015;35:1439-42.

43. Chuckpaiwong B, Charles HC, Kraus VB, Guilak F, Nunley JA. Age-associated increases in the size of the infrapatellar fat pad in knee osteoarthritis as measured by 3T MRI. J Orthop Res 2010;28:1149-54.

44. Collins NJ, Vicenzino B, van der Heijden RA, van Middelkoop M. Pain during prolonged sitting is a common problem in persons with patellofemoral pain. J Orthop Sports Phys Ther 2016;46:658-63.

45. Näslund J, Waldén M, Lindberg LG. Decreased pulsatile blood flow in the patella in patellofemoral pain syndrome. Am J Sports Med 2007;35:1668-73.

46. Tiderius CJ, Tjornstrand J, Akeson P, Sodersten K, Dahlberg L, Leander P. Delayed gadolinium-enhanced MRI of cartilage (dGEMRIC): Intra- and interobserver variability in standardized drawing of regions of interest. Acta Radiol 2004;45:628-34. 


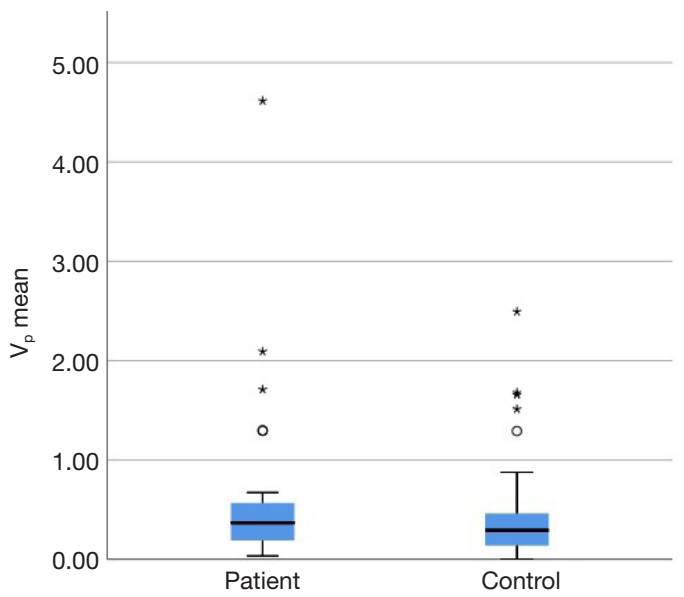

Figure S1 Boxplot of $V_{p}$ multiplied by 1,000 with exclusion of the biggest patient outlier. The * means the remaining outliers. 